\title{
Immunomodulatory Effects of Macrolide Antibiotics - Part 1: Biological Mechanisms
}

\author{
J. Altenburg ${ }^{a} \quad$ C.S. de Graaffa ${ }^{a}$ T.S. van der Werf ${ }^{b} \quad$ W.G. Boersma ${ }^{a}$ \\ a Department of Pulmonary Diseases, Medical Centre Alkmaar, Alkmaar, and b Department of Internal Medicine, \\ University Medical Centre Groningen, University of Groningen, Groningen, The Netherlands
}

\section{Key Words}

Diffuse panbronchiolitis · Macrolides · Quorum sensing

\begin{abstract}
Macrolide antibiotics are well known for their antibacterial and anti-inflammatory properties. This article provides an overview of the biological mechanisms through which macrolides exert this 'double effect'. Their antibacterial effect consists of the inhibition of bacterial protein synthesis, impaired bacterial biofilm synthesis, and the attenuation of other bacterial virulence factors. Apart from these direct antimicrobial effects, macrolides are known for their modulating effect on many components of the human immune system. By influencing the production of cytokines, they have a dampening effect on the proinflammatory response. Furthermore, the majority of cells involved in the immune response are, in one way or another, influenced when macrolide antibiotics are administered. Having such an obvious effect on the various aspects of the immune system, macrolides seem to be exceptionally suited for the treatment of chronic inflammatory diseases.

Copyright $\odot 2010$ S. Karger AG, Basel
\end{abstract}

\section{Introduction}

Since their discovery in 1952, many beneficial effects have been attributed to antibiotics belonging to the macrolide family, originally isolated from cultures of Streptomyces erythraea. Macrolide antibiotics were named after their main characteristic: a macrocyclic lactone ring which can contain up to 23 atoms [1]. The most commonly used macrolides have 14 (e.g. erythromycin, clarithromycin, and roxithromycin) or 15 (e.g. azithromycin) atoms attached to their macrocyclic rings and are therefore defined as 14- or 15-membered ring macrolides. Over the last decades macrolide antibiotics have been used as a treatment for common infectious diseases like pneumonia, bronchitis, pharyngitis, and skin infections, possessing a moderately broad spectrum of antibacterial activity.

An accumulating body of evidence has emerged indicating that 14- and 15-membered ring macrolides possess modes of action which are independent of their antimicrobial activity. This first became known in 1987 when Kudoh et al. [2] reported a spectacular decrease in symptoms and an increase in life expectancy in patients with diffuse panbronchiolitis (DPB) when they were treated with the macrolide erythromycin. Until then, DPB had been a rapidly progressive and debilitating inflammatory

Drs. J. Altenburg, MD

Department of Pulmonary Diseases

Medical Centre Alkmaar, Wilhelminalaan 12

NL-1812 JD Alkmaar (The Netherlands)

Tel. +31 72548 2750, Fax +31 72548 2167, E-Mail j.altenburg@mca.nl 
airway disorder associated with a very poor prognosis. After 1987, when erythromycin was introduced as standard therapy for DPB, an impressive increase in 10-year survival was seen, i.e. from $10-20 \%$ to over $90 \%$ [3-6].

The unexpected success was attributed to a previously unknown anti-inflammatory effect of erythromycin. This theory was supported by the fact that serum levels of erythromycin in these DPB patients were well below minimal inhibitory concentrations (MIC) for the detected pathogens and by the known lack of susceptibility of most Gram-negative organisms to erythromycin.

In the last two decades, exhaustive evidence has shown that macrolides indeed have a direct antimicrobial effect but, more importantly, also modulate many components of the immune response. Because of this anti-inflammatory or 'immune modulating' effect, macrolide antibiotics have been widely used as maintenance treatment for various chronic inflammatory pulmonary diseases. Chronic inflammatory diseases generally feature a distorted inflammatory response. Instead of protecting the human body against exogenous attacks, the cascade of anti-inflammatory responses fails, damaging cells and making them more vulnerable to new attacks. In this article we aim to clarify the biological mechanisms through which macrolides exert their immunemodulating and antibacterial effect. These mechanisms are shown schematically in figure 1 .

\section{Effects on Host-Pathogen Interactions}

Most macrolides are active against Gram-positive cocci (including anaerobes) and have limited Gram-negative activity. They inhibit bacterial protein synthesis by binding to the $50 \mathrm{~S}$ subunit of the ribosome $[1,7,8]$.

\section{Biofilm}

A biofilm is an aggregate of microorganisms immersed in a polysaccharide matrix adherent to each other and to the airway mucosa. Biofilm-forming bacteria are protected from phagocytosis, antimicrobial agents, and the ciliary action of the airway epithelial cells. Furthermore, microorganisms gathered in a biofilm develop significantly different genetic properties compared to planktonic species. Research on the biofilm effects of macrolides mainly focuses on Pseudomonas aeruginosa, which is one of the more virulent biofilm-forming microorganisms with a natural resistance to macrolides.

However, effects of macrolides were also demonstrated on biofilm formation in Haemophilus influenzae and
Staphylococcus epidermidis $[9,10]$. Macrolides have been shown to alter the structure and architecture of the bacterial biofilm [11-13]. Results of Japanese in vitro studies indicate that azithromycin and clarithromycin change the structure of bacterial biofilms via the inhibition of polysaccharide synthesis $[12,14]$. An insufficient biofilm allows for enhanced phagocytosis and clearance of bacteria by alveolar macrophages $[11,15]$.

\section{Quorum Sensing}

During infection, bacteria employ mutual communication [quorum sensing (QS)] to coordinate the expression of genes, for instance, genes encoding for tissuedamaging factors [16]. Through the production of autoinducer molecules, genes can be switched on or off depending on the local pathogen density. Furthermore, activation of the QS cascade is claimed to promote biofilm formation and to stimulate IL- 8 production, causing an enhanced neutrophil influx at the site of infection [6]. Several authors suggest that suppression of QS systems through reduced transcription of QS genes is also one of the mechanisms of macrolide action [1618].

\section{Bacterial Adherence}

In vitro and in vivo evidence suggests that $P$. aeruginosa bacilli, when cultured in the presence of low levels of macrolides, e.g. erythromycin, demonstrate decreased adherence to cells of the airway epithelium [19-21]. Since the adherence of bacteria to mucosal surfaces is an important initial event in the pathogenesis of most bacterial infectious diseases, this could help explain the clinical efficacy of low-dose macrolide therapy in patients colonized with PA.

\section{Mobility}

The effect of macrolides on P. aeruginosa is accompanied by an impairment of the mobility of this microorganism. Pseudomonas spp. are mobile thanks to 2 distinctive modalities: flagella, which are tail-like structures that project from the cell body and move in a whip-like manner, and type IV pili (fimbriae) that provide twitching motility.

Exposure to sub-MIC concentrations of macrolide antibiotics results in loss of mobility, partly due to the inhibition of flagellin production [22-24], the principal constituent of bacterial flagella, and partly because some macrolides alter the assembly of type IV pili $[13,25]$. This loss of mobility facilitates easier phagocytosis and the killing of bacteria by alveolar macrophages. 


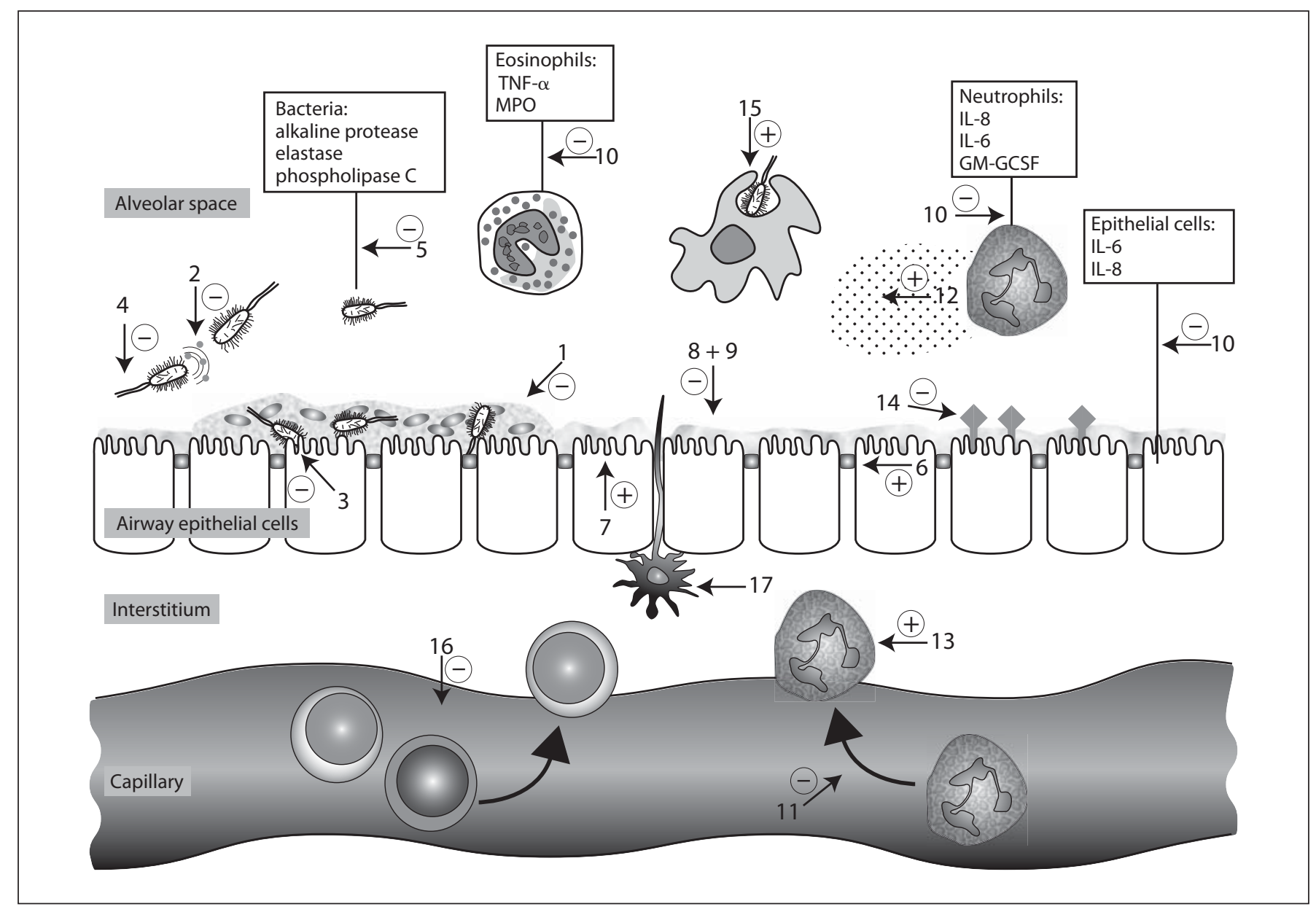

Fig. 1. Cellular and noncellular effects of macrolides. $1=$ Attenuation of biofilm function; 2 = suppression of bacterial quorum sensing; 3 = decrease in bacterial adherence; 4 = loss of flagellar mobility; 5 = reduced production of bacterial toxins; $6=$ consolidation of epithelial tight junctions; $7=$ increasing ciliary beat frequency; $8=$ reduction of sputum quantity; $9=$ diminished sputum viscosity; 10 inhibition of the synthesis of proinflammatory agents by bacteria, eosinophils, neutrophils, and epithelial cells; 11 = reduction of neutrophil chemotaxis; 12 = stimulation of neutrophil degranulation; 13 = acceleration of neutrophil apoptosis; 14 = downregulation of adhesion molecules; 15 = stimulation of phagocytosis by alveolar macrophages; 16 = reduction of $\mathrm{T}$ cell numbers and $\mathrm{T}$ cell migration; 17 = modulation of dendritic cell function.

\section{Bacterial Toxins}

Cytotoxic enzymes produced by bacteria when causing infection, including exotoxin A, alkaline protease, elastase, and phospholipase $\mathrm{C}$, are important factors in bacterial virulence. Erythromycin and, more recently, azithromycin have been shown to suppress the production of those enzymes and, consequently, to diminish bacterial virulence $[23,26-28]$.

\section{Intracellular Effects}

Macrolides accumulate and show a prolonged retention in human cells after oral or intravenous administra- tion, an effect that is augmented when macrolide treatment is given for a longer period of time [29-32]. In cystic fibrosis patients treated with azithromycin $(500 \mathrm{mg}$ daily) for at least 35 consecutive days, the concentration of azithromycin in neutrophils appeared to be up to 3,000 times higher as compared to the concentration in plasma [32]. Macrolides have also been shown to accumulate in alveolar macrophages [30, 33].

This suggests that tissue and intracellular concentrations may be more useful for assessing the antibacterial activity of azitromycin than serum concentrations [34, 35]. Because intracellular concentrations of macrolide 
antibiotic often exceed the MIC of phagocytized pathogens, macrolides have also been demonstrated to be effective against microorganisms with in vitro macrolide resistance $[35,36]$. The excellent intracellular penetration of macrolides also appears to explain their effectiveness against intracellular pathogens [34].

\section{Effects on Airway Epithelial Cells and Mucus Properties}

Besides inhibiting the production of proinflammatory cytokines by bronchial epithelial cells $[37,38]$, macrolides distinctly modulate features of the bronchial epithelium, making it better armed against exogenous attacks. The bronchial epithelium is critically important in lung defense. In addition to being a mechanical barrier, it regulates the electrolyte content of the airway surface liquid by means of its tight junctions between adjacent cells. In vitro studies demonstrate that azithromycin increases the transepithelial electrical resistance of human airway epithelium by changing the processing of tight junction proteins, thus preventing excess leakage of electrolytes and ameliorating mucus properties [39].

Furthermore, when airway epithelial cells are exposed to inflammatory mediators in vitro, macrolides display a protective effect against epithelial damage and ciliary dysfunction $[40,41]$. This positive effect on ciliary beat frequency, however, was not confirmed in in vivo studies in patients with chronic rhinosinusitis or bronchiectasis $[42,43]$.

Airway mucus hypersecretion and the resulting excess sputum expectoration is an important characteristic of several chronic inflammatory pulmonary diseases. $\mathrm{Mu}-$ cus hypersecretion may lead to more exacerbations and poor health-related quality of life [44]. Macrolides have been shown not only to reduce the quantity of expectorated sputum in vivo, e.g. in bronchiectasis, but also to change the composition of mucus, thereby enhancing mucus clearance [45-51].

\section{Effects on the Immune System}

\section{Innate Immunity}

Cytokine and Chemokine Response

Cytokines are hormone-like proteins that enable immune cells to communicate, and they play an integral role in the initiation, perpetuation, and subsequent downregulation of the immune response. Chemokines are cyto- kines with a particularly strong chemotactic capacity. Production of cytokines is effectuated by a variety of cell types, including alveolar macrophages, eosinophils, neutrophils, and bronchial epithelial cells. Proinflammatory cytokines [such as interleukin (IL)-1, IL-2, IL-4, IL-6, IFN- $\gamma$, TNF- $\alpha$, and GM-GCSF) and chemokines (such as IL- 8 and RANTES) amplify the immune response through positive feedback loops. Anti-inflammatory cytokines, such as IL-10, prostaglandins, and transforming growth factor (TGF)- $\beta$, attenuate the immune response through a negative-feedback mechanism. In general, macrolides inhibit the synthesis and/or secretion of proinflammatory cytokines while increasing the release of antiinflammatory cytokines [1]. Some recent research however, promotes a view in which macrolides can differentially modulate proinflammatory cytokine secretion [37]. Changes in cytokine and chemokine production are probably related to an effect of macrolides on the activation of transcription factors, i.e. nuclear factor (NF)- $\kappa B$ and activator protein (AP)-1 [52]. Inhibition of the production of proinflammatory cytokines has been described in several in vivo studies in healthy subjects and in patients with CF, asthma, or chronic rhinosinusitis [53-59].

\section{Alveolar Macrophages}

Macrophages play a critical role in the phagocytosis of apoptotic cells and in the removal of exogenous particles, such as bacteria. Recent studies prove that macrolides promote the phagocytosis of apoptotic cells by alveolar macrophages, thus avoiding secondary necrosis and the release of cell contents that may induce further inflammation [60-62].

In addition, some authors propose that macrolides promote monocyte-to-macrophage differentiation, thereby increasing the number of active macrophages [63, 64]. Results of earlier research suggest that macrolide antibiotics also enhance other macrophage functions, including cytocidal activity [65].

\section{Neutrophils}

Neutrophils are key players in the inflammatory response of patients with chronic airway disease [66]. They accumulate at the site of infection, responding to increased levels of chemokines and cytokines, primarily IL-8 and TNF- $\alpha$. Macrolide antibiotics exert an influence on several domains of neutrophil function.

Reaction to Chemokines. Macrolide antibiotics cause a significant reduction in the chemotactic response of neutrophils to chemokines $[67,68]$. Together with the previously described inhibition of chemoattractant genera- 
tion, this results in markedly decreased airway neutrophilia in patients with various inflammatory pulmonary diseases [8, 51, 59, 69-72].

Degranulation. Upon activation, neutrophils release granules containing cytotoxic enzymes, such as elastase, a process called neutrophil degranulation or exocytosis. In general, macrolides seem to stimulate exocytosis, which may result in enhanced antibactericidal activity $[1$, 73-76].

Adhesion. Leukocyte adhesion is a hallmark of the inflammatory cascade, and cell adhesion molecules are the mediators of this event [1]. Cultured bronchial epithelial cells treated with erythromycin show reduced levels of intercellular adhesion molecule $1[38,77]$. These findings suggest that reducing the release of adhesion molecules in bronchial epithelial cells is another anti-inflammatory effect of macrolide antibiotics.

Oxidative Burst. The production and release of reactive oxygen species by neutrophils to enhance their cytotoxic capability is referred to as the 'oxidative' or 'respiratory' burst, a process mediated by NADPH-dependent oxidase. Contradictory data have been reported with regard to the effect of macrolides on the oxidative burst. Initially, evidence was presented showing an attenuation of the oxidative burst capacity, but more recent studies have disclosed an opposite effect or no effect at all [74, 78-80].

Apoptosis. In the previous decade, it had already been proposed that apoptosis (programmed cell death) limits the ability of neutrophils to damage tissue while being involved in an inflammatory response [81, 82]. Since then, several in vitro studies have demonstrated that macrolides shorten neutrophil survival by accelerating neutrophil apoptosis [74, 79, 82-84].

\section{Adaptive Immunity}

The aforementioned research data indisputably show the existence of a direct modulating effect of macrolides on the innate immune system. Studies focusing on the effects of macrolide antibiotics on cellular immunity also clearly demonstrate an impact on $\mathrm{T}$ cell regulation and antigen presentation.

Long-term use of macrolide antibiotics reduces the elevated number of lymphocytes in the bronchoalveolar lavage fluid of DPB patients to subnormal levels $[85,86]$. In addition, 14- and 15-membered ring macrolides appear to be involved in the augmentation of the apoptosis of activated lymphocytes, thus reducing inflammation [87]. Dendritic cells are the most important antigen-presenting cells and play a central role in the initiation and regu- lation of immune responses. Sugiyama et al. [88] demonstrated that clarithromycin and azithromycin modulate the function of dendritic cells; each macrolide shows a different immune-dampening effect. In addition, macrolides appear to have a suppressive effect on the proinflammatory cytokine production of $\mathrm{T}$ cells $[89,90]$.

An early in vivo study in healthy volunteers showed a small but significant positive effect of azithromycin on the proliferative B cell response of stimulated lymphocytes [91]. However, a more recent study in patients with bronchiectasis failed to confirm this finding [92], while research in vitro demonstrated an opposite effect [93].

\section{Conclusion}

Macrolide antibiotics are well known for their antibacterial and anti-inflammatory properties. They clearly posses an antibacterial effect that consists of the inhibition of bacterial protein synthesis, impaired bacterial biofilm synthesis, and the attenuation of other bacterial virulence factors. Apart from these direct antimicrobial effects, macrolides are known for their modulating effect on many components of the human immune system. By influencing the production of cytokines, they have a dampening effect on the proinflammatory response. Furthermore, the majority of the cells involved in both the innate and adaptive immune responses are, in one way or another, influenced when macrolide antibiotics are administered. The most distinct effect of macrolides is found in neutrophils, the key players of the anti-inflammatory response. Among other things, neutrophil accumulation, adhesion, and apoptosis are clearly reduced, which results in markedly decreased airway neutrophilia. Studies focusing on the effects of macrolide antibiotics on cellular immunity also clearly demonstrate an impact on $\mathrm{T}$ cell regulation and antigen presentation.

\section{Future Perspectives}

In the near future, clinicians might add new immunomodulatory drugs of the macrolide family to their armamentarium. Immunomodulatory macrolide antibiotics devoid of anti-infective properties are developed by modifying the molecular structure of the atoms attached to the macrocyclic ring. These purely immunomodulatory macrolides would offer a way to circumvent bacterial resistance. This concept has been investigated in tetracyclines, another group of antibiotics which also have 
anti-inflammatory properties. Chemically modified tetracyclines, with no antibacterial capacity, induce an antiinflammatory response by modulating cy tokine and matrix metalloproteinase secretion [94-98]. However, only in vitro and animal studies have been performed investigating the effect of chemically modified tetracyclines. To our knowledge, no phase 1 studies are yet available describing the efficacy and safety of purely immunomodulatory drugs.

\section{Acknowledgement}

We would like to thank Mr. Rene Blom for creating the illustration.

\section{References}

1 Culic O, Erakovic V, Parnham MJ: Anti-inflammatory effects of macrolide antibiotics. Eur J Pharmacol 2001;429:209-229.

$\checkmark 2$ Kudoh S, Uetake T, Hagiwara K: Clinical effects of low-dose long-term erythromycin chemotherapy on diffuse panbronchiolitis (in Japanese). Nihon Kyobu Shikkan Gakkai Zasshi 1987;25:632-642.

3 Fujii T, Kadota J, Kawakami K: Long term effect of erythromycin therapy in patients with chronic Pseudomonas aeruginosa infection. Thorax 1995;50:1246-1252.

4 Kudoh S, Azuma A, Yamamoto M: Improve ment of survival in patients with diffuse panbronchiolitis treated with low-dose erythromycin. Am J Respir Crit Care Med 1998;157: 1829-1832.

5 Nagai H, Shishido H, Yoneda R: Long-term low-dose administration of erythromycin to patients with diffuse panbronchiolitis. Respiration 1991;58:145-149.

6 Tateda K, Ishii Y, Kimura S: Suppression of Pseudomonas aeruginosa quorum-sensing systems by macrolides: a promising strategy or an oriental mystery? J Infect Chemother 2007;13:357-367.

7 Healy DP: Macrolide immunomodulation of chronic respiratory diseases. Curr Infect Dis Rep 2007;9:7-13.

$\checkmark 8$ Idris SF, Chilvers ER, Haworth C: Azithromycin therapy for neutrophilic airways disease: myth or magic? Thorax 2009;64:186189.

-9 Starner TD, Shrout JD, Parsek MR: Subinhibitory concentrations of azithromycin decrease nontypeable Haemophilus influenzae biofilm formation and diminish established biofilms. Antimicrob Agents Chemother 2008;52:137-145.

10 Yasuda H, Ajiki Y, Koga T: Interaction between clarithromycin and biofilms formed by Staphylococcus epidermidis. Antimicrob Agents Chemother 1994;38:138-141.

11 Ichimiya T, Yamasaki T, Nasu M: In-vitro effects of antimicrobial agents on Pseudomonas aeruginosa biofilm formation. J Antimicrob Chemother 1994;34:331-341.
12 Ichimiya T, Takeoka K, Hiramatsu K: The influence of azithromycin on the biofilm formation of Pseudomonas aeruginosa in vitro. Chemotherapy 1996;42:186-191.

13 Wozniak DJ, Keyser R: Effects of subinhibitory concentrations of macrolide antibiotics on Pseudomonas aeruginosa. Chest 2004; 125:62S-69S.

14 Kondoh K, Hashiba M, Baba S: Inhibitory activity of clarithromycin on biofilm synthesis with Pseudomonas aeruginosa. Acta Otolaryngol Suppl 1996;525:56-60.

15 Takeoka K, Ichimiya T, Yamasaki T: The in vitro effect of macrolides on the interaction of human polymorphonuclear leukocytes with Pseudomonas aeruginosa in biofilm. Chemotherapy 1998;44:190-197.

16 Skindersoe ME, Alhede M, Phipps R: Effects of antibiotics on quorum sensing in Pseudomonas aeruginosa. Antimicrob Agents Chemother 2008;52:3648-3663.

17 Nalca Y, Jansch L, Bredenbruch F: Quorumsensing antagonistic activities of azithromycin in Pseudomonas aeruginosa PAO1: a global approach. Antimicrob Agents Chemother 2006;50:1680-1688.

18 Tateda K, Comte R, Pechere JC: Azithromycin inhibits quorum sensing in Pseudomonas aeruginosa. Antimicrob Agents Chemother 2001;45:1930-1933.

19 Baumann U, Fischer JJ, Gudowius P: Buccal adherence of Pseudomonas aeruginosa in patients with cystic fibrosis under long-term therapy with azithromycin. Infection 2001; 29:7-11.

20 Tsang KW, Ng P, Ho PL: Effects of erythromycin on Pseudomonas aeruginosa adherence to collagen and morphology in vitro. Eur Respir J 2003;21:401-406.

-21 Yamasaki T: Adherence of Pseudomonas aeruginosa to mouse tracheal epithelium - the effect of antimicrobial agents (in Japanese). Kansenshogaku Zasshi 1990;64:575-583.

22 Molinari G, Paglia P, Schito GC: Inhibition of motility of Pseudomonas aeruginosa and Proteus mirabilis by subinhibitory concentrations of azithromycin. Eur J Clin Microbiol Infect Dis 1992;11:469-471.
23 Molinari G, Guzman CA, Pesce A: Inhibition of Pseudomonas aeruginosa virulence factors by subinhibitory concentrations of azithromycin and other macrolide antibiotics. J Antimicrob Chemother 1993;31:681-688.

24 Kawamura-Sato K, Iinuma Y, Hasegawa T: Effect of subinhibitory concentrations of macrolides on expression of flagellin in Pseudomonas aeruginosa and Proteus mirabilis. Antimicrob Agents Chemother 2000; 44:2869-2872.

25 Hentzer M, Givskov M: Pharmacological inhibition of quorum sensing for the treatment of chronic bacterial infections. J Clin Invest 2003;112:1300-1307.

26 Hirakata Y, Kaku M, Mizukane R: Potential effects of erythromycin on host defense systems and virulence of Pseudomonas aeruginosa. Antimicrob Agents Chemother 1992; 36:1922-1927.

27 Kita E, Sawaki M, Oku D: Suppression of virulence factors of Pseudomonas aeruginosa by erythromycin. J Antimicrob Chemother 1991;27:273-284.

28 Mizukane R, Hirakata Y, Kaku M: Comparative in vitro exoenzyme-suppressing activities of azithromycin and other macrolide antibiotics against $P$ seudomonas aeruginosa. Antimicrob Agents Chemother 1994;38:528-533.

-29 Bosnar M, Kelneric Z, Munic V: Cellular uptake and efflux of azithromycin, erythromycin, clarithromycin, telithromycin, and cethromycin. Antimicrob Agents Chemother 2005;49:2372-2377.

30 Capitano B, Mattoes HM, Shore E: Steadystate intrapulmonary concentrations of moxifloxacin, levofloxacin, and azithromycin in older adults. Chest 2004;125:965-973.

- 31 Wildfeuer A, Laufen H, Zimmermann T: Distribution of orally administered azithromycin in various blood compartments. Int J Clin Pharmacol Ther 1994;32:356-360.

32 Wilms EB, Touw DJ, Heijerman HG: Pharmacokinetics of azithromycin in plasma, blood, polymorphonuclear neutrophils and sputum during long-term therapy in patients with cystic fibrosis. Ther Drug Monit 2006; 28:219-225. 
33 Lucchi M, Damle B, Fang A: Pharmacokinetics of azithromycin in serum, bronchial washings, alveolar macrophages and lung tissue following a single oral dose of extended or immediate release formulations of azithromycin. J Antimicrob Chemother 2008;61:884-891

-34 Nakamura S, Yanagihara K, Araki N: Efficacy of clarithromycin against experimentally induced pneumonia caused by clarithromycin-resistant Haemophilus influen$z a e$ in mice. Antimicrob Agents Chemother 2010;54:757-762.

-35 Yanagihara K, Izumikawa K, Higa F: Efficacy of azithromycin in the treatment of community-acquired pneumonia, including patients with macrolide-resistant Streptococcus pneumoniae infection. Intern Med 2009;48: 527-535.

-36 Hoffman HL, Klepser ME, Ernst EJ: Influence of macrolide susceptibility on efficacies of clarithromycin and azithromycin against Streptococcus pneumoniae in a murine lung infection model. Antimicrob Agents Chemother 2003;47:739-746.

>37 Shinkai M, Foster GH, Rubin BK: Macrolide antibiotics modulate ERK phosphorylation and IL- 8 and GM-CSF production by human bronchial epithelial cells. Am J Physiol Lung Cell Mol Physiol 2006;290:L75-L85.

>38 Khair OA, Devalia JL, Abdelaziz MM: Effect of erythromycin on Haemophilus influenzae endotoxin-induced release of IL-6, IL-8 and sICAM-1 by cultured human bronchial epithelial cells. Eur Respir J 1995;8:1451-1457.

>39 Asgrimsson V, Gudjonsson T, Gudmundsson GH: Novel effects of azithromycin on tight junction proteins in human airway epithelia. Antimicrob Agents Chemother 2006; 50:1805-1812.

$\checkmark 40$ Feldman C, Anderson R, Theron AJ: Roxithromycin, clarithromycin, and azithromycin attenuate the injurious effects of bioactive phospholipids on human respiratory epithelium in vitro. Inflammation 1997;21: 655-665.

-41 Anderson R, Theron AJ, Feldman C: Membrane-stabilizing, anti-inflammatory interactions of macrolides with human neutrophils. Inflammation 1996;20:693-705.

-42 Cervin A, Kalm O, Sandkull P: One-year low-dose erythromycin treatment of persistent chronic sinusitis after sinus surgery: clinical outcome and effects on mucociliary parameters and nasal nitric oxide. Otolaryngol Head Neck Surg 2002;126:481-489.

43 Shibuya Y, Wills PJ, Cole PJ: The effect of erythromycin on mucociliary transportability and rheology of cystic fibrosis and bronchiectasis sputum. Respiration 2001;68:615619.

-44 Bhowmik A, Chahal K, Austin G: Improving mucociliary clearance in chronic obstructive pulmonary disease. Respir Med 2009; 103:496-502.
45 Cymbala AA, Edmonds LC, Bauer MA: The disease-modifying effects of twice-weekly oral azithromycin in patients with bronchiectasis. Treat Respir Med 2005;4:117-122.

46 Davies G, Wilson R: Prophylactic antibiotic treatment of bronchiectasis with azithromycin. Thorax 2004;59:540-541.

47 Rubin BK, Druce H, Ramirez OE: Effect of clarithromycin on nasal mucus properties in healthy subjects and in patients with purulent rhinitis. Am J Respir Crit Care Med 1997;155:2018-2023.

48 Shirai T, Sato A, Chida K: Effect of 14-membered ring macrolide therapy on chronic respiratory tract infections and polymorphonuclear leukocyte activity. Intern Med 1995; 34:469-474

49 Tagaya E, Tamaoki J, Kondo M: Effect of a short course of clarithromycin therapy on sputum production in patients with chronic airway hypersecretion. Chest 2002;122:213218.

50 Tsang KW, Ho PI, Chan KN: A pilot study of low-dose erythromycin in bronchiectasis. Eur Respir J 1999;13:361-364.

51 Yalcin E, Kiper N, Ozcelik U: Effects of claritromycin on inflammatory parameters and clinical conditions in children with bronchiectasis. J Clin Pharm Ther 2006;31: 49-55.

52 Desaki M, Takizawa H, Ohtoshi T: Erythromycin suppresses nuclear factor-kappaB and activator protein-1 activation in human bronchial epithelial cells. Biochem Biophys Res Commun 2000;267:124-128.

53 Kurdowska A, Noble JM, Griffith DE: The effect of azithromycin and clarithromycin on ex vivo interleukin-8 (IL-8) release from whole blood and IL- 8 production by human alveolar macrophages. J Antimicrob Chemother 2001;47:867-870.

54 Equi A, Balfour-Lynn IM, Bush A: Long term azithromycin in children with cystic fibrosis: a randomised, placebo-controlled crossover trial. Lancet 2002;360:978-984.

55 Kraft M, Cassell GH, Pak J: Mycoplasma pneumoniae and Chlamydia pneumoniae in asthma: effect of clarithromycin. Chest 2002;121:1782-1788.

56 Takizawa H, Desaki M, Ohtoshi T: Erythromycin and clarithromycin attenuate cytokine-induced endothelin-1 expression in human bronchial epithelial cells. Eur Respir J 1998;12:57-63.

57 Wallwork B, Coman W, Mackay-Sim A: A double-blind, randomized, placebo-controlled trial of macrolide in the treatment of chronic rhinosinusitis. Laryngoscope 2006; 116:189-193

58 Yamada T, Fujieda S, Mori S: Macrolide treatment decreased the size of nasal polyps and IL-8 levels in nasal lavage. Am J Rhinol 2000; $14: 143-148$.

59 Simpson JL, Powell H, Boyle MJ: Clarithromycin targets neutrophilic airway inflammation in refractory asthma. Am J Respir Crit Care Med 2008;177:148-155.
60 Hodge S, Hodge G, Brozyna S: Azithromycin increases phagocytosis of apoptotic bronchial epithelial cells by alveolar macrophages. Eur Respir J 2006;28:486-495.

61 Yamaryo T, Oishi K, Yoshimine H: Fourteen-member macrolides promote the phosphatidylserine receptor-dependent phagocytosis of apoptotic neutrophils by alveolar macrophages. Antimicrob Agents Chemother 2003;47:48-53.

62 Hodge S, Hodge G, Jersmann H: Azithromycin improves macrophage phagocytic function and expression of mannose receptor in chronic obstructive pulmonary disease. Am J Respir Crit Care Med 2008;178:139-148.

63 Keicho N, Kudoh S, Yotsumoto H: Erythromycin promotes monocyte to macrophage differentiation. J Antibiot (Tokyo) 1994;47: 80-89.

64 Yoshida K, Sunazuka T, Nagai K: Macrolides with promotive activity of monocyte to macrophage differentiation. J Antibiot (Tokyo) 2005;58:79-81.

65 Xu G, Fujita J, Negayama K: Effect of macrolide antibiotics on macrophage functions. Microbiol Immunol 1996;40:473-479.

66 Tamaoki J, Kadota J, Takizawa H: Clinical implications of the immunomodulatory effects of macrolides. Am J Med 2004;117(suppl 9A):5S-11S.

-67 Tamaoki J, Nakata J, Tagaya E: Effects of roxithromycin and erythromycin on interleukin 8-induced neutrophil recruitment and goblet cell secretion in guinea pig tracheas. Antimicrob Agents Chemother 1996;40: 1726-1728.

68 Tsai WC, Rodriguez ML, Young KS: Azithromycin blocks neutrophil recruitment in Pseudomonas endobronchial infection. Am J Respir Crit Care Med 2004;170:1331-1339.

-69 Piacentini GL, Peroni DG, Bodini A: Azithromycin reduces bronchial hyperresponsiveness and neutrophilic airway inflammation in asthmatic children: a preliminary report. Allergy Asthma Proc 2007;28: 194-198.

70 Sakito O, Kadota J, Kohno S: Interleukin 1 beta, tumor necrosis factor alpha, and interleukin 8 in bronchoalveolar lavage fluid of patients with diffuse panbronchiolitis: a potential mechanism of macrolide therapy. Respiration 1996;63:42-48.

71 Suzuki H, Shimomura A, Ikeda K: Effects of long-term low-dose macrolide administration on neutrophil recruitment and IL-8 in the nasal discharge of chronic sinusitis patients. Tohoku J Exp Med 1997;182:115-124.

72 Verleden GM, Vanaudenaerde BM, Dupont LJ: Azithromycin reduces airway neutrophilia and interleukin- 8 in patients with bronchiolitis obliterans syndrome. Am J Respir Crit Care Med 2006;174:566-570.

73 Abdelghaffar H, Vazifeh D, Labro MT: Comparison of various macrolides on stimulation of human neutrophil degranulation in vitro. J Antimicrob Chemother 1996;38:81-93. 
74 Culic O, Erakovic V, Cepelak I: Azithromycin modulates neutrophil function and circulating inflammatory mediators in healthy human subjects. Eur J Pharmacol 2002;450: 277-289.

-75 Labro MT, el Benna J, Babin-Chevaye C: Comparison of the in-vitro effect of several macrolides on the oxidative burst of human neutrophils. J Antimicrob Chemother 1989; 24:561-572.

76 Vazifeh D, Preira A, Bryskier A: Interactions between HMR 3647, a new ketolide, and human polymorphonuclear neutrophils. Antimicrob Agents Chemother 1998;42:19441951.

-77 Tamaoki J: The effects of macrolides on inflammatory cells. Chest 2004;125:41S-50S.

78 Hand WL, Hand DL, King-Thompson NL: Antibiotic inhibition of the respiratory burst response in human polymorphonuclear leukocytes. Antimicrob Agents Chemother 1990;34:863-870.

-79 Koch CC, Esteban DJ, Chin AC: Apoptosis, oxidative metabolism and interleukin-8 production in human neutrophils exposed to azithromycin: effects of Streptococcus pneumoniae. J Antimicrob Chemother 2000;46: 19-26.

80 Levert H, Gressier B, Brunet C: Time and concentration dependent influence of dirithromycin on neutrophils oxidative burst. J Antibiot (Tokyo) 1999;52:127-133.

-81 Whyte MK, Meagher LC, MacDermot J: Impairment of function in aging neutrophils is associated with apoptosis. J Immunol 1993; 150:5124-5134.
82 Aoshiba K, Nagai A, Konno K: Erythromycin shortens neutrophil survival by accelerating apoptosis. Antimicrob Agents Chemother 1995;39:872-877

-83 Inamura K, Ohta N, Fukase S: The effects of erythromycin on human peripheral neutrophil apoptosis. Rhinology 2000;38:124-129.

84 Yamasawa H, Oshikawa K, Ohno S: Macrolides inhibit epithelial cell-mediated neutrophil survival by modulating granulocyte macrophage colony-stimulating factor release. Am J Respir Cell Mol Biol 2004;30: 569-575.

85 Kawakami K, Kadota J, Iida K: Phenotypic characterization of $\mathrm{T}$ cells in bronchoalveolar lavage fluid (BALF) and peripheral blood of patients with diffuse panbronchiolitis: the importance of cytotoxic T cells. Clin Exp Immunol 1997;107:410-416.

86 Mukae H, Kadota J, Kohno S: Increase in activated CD8+ cells in bronchoalveolar lavage fluid in patients with diffuse panbronchiolitis. Am J Respir Crit Care Med 1995;152: 613-618.

87 Kadota J, Mizunoe S, Kishi K: Antibiotic-induced apoptosis in human activated peripheral lymphocytes. Int J Antimicrob Agents 2005;25:216-220.

88 Sugiyama K, Shirai R, Mukae H: Differing effects of clarithromycin and azithromycin on cytokine production by murine dendritic cells. Clin Exp Immunol 2007;147:540-546.

89 Asano K, Kamakazu K, Hisamitsu T: Modulation of Th2 type cytokine production from human peripheral blood leukocytes by a macrolide antibiotic, roxithromycin, in vitro. Int Immunopharmacol 2001;1:19131921.

$\checkmark 90$ Williams AC, Galley HF, Watt AM: Differential effects of three antibiotics on T helper cell cytokine expression. J Antimicrob Chemother 2005;56:502-506.
91 Tomazic J, Kotnik V, Wraber B: In vivo administration of azithromycin affects lymphocyte activity in vitro. Antimicrob Agents Chemother 1993;37:1786-1789.

92 Harita S, Kuyama S, Okada T: Effect of longterm and low-dose administration of erythromycin on proliferation of $\mathrm{T}$ lymphocytes stimulated with mitogens. J Chemother 2008;20:604-608.

$\checkmark 93$ Keicho N, Kudoh S, Yotsumoto H: Antilymphocytic activity of erythromycin distinct from that of FK506 or cyclosporin A. J Antibiot (Tokyo) 1993;46:1406-1413.

94 Cazalis J, Tanabe S, Gagnon G: Tetracyclines and chemically modified tetracycline-3 (CMT-3) modulate cytokine secretion by lipopolysaccharide-stimulated whole blood. Inflammation 2009;32:130-137.

$\checkmark 95$ Golub LM, Suomalainen K, Sorsa T: Host modulation with tetracyclines and their chemically modified analogues. Curr Opin Dent 1992;2:80-90.

$>96$ Maisi P, Kiili M, Raulo SM: MMP inhibition by chemically modified tetracycline- 3 (CMT-3) in equine pulmonary epithelial lining fluid. Ann NY Acad Sci 1999;878:675677.

$\checkmark 97$ Maitra SR, Bhaduri S, Chen E: Role of chemically modified tetracycline on TNF-alpha and mitogen-activated protein kinases in sepsis. Shock 2004;22:478-481.

$>98$ Steinberg J, Halter J, Schiller H: Chemically modified tetracycline prevents the development of septic shock and acute respiratory distress syndrome in a clinically applicable porcine model. Shock 2005;24:348-356. 